\title{
Dispositivos de cierre percutáneo de orejuela para la prevención de embolia en fibrilación auricular
}

\section{Devices for percutaneous appendage occlusion in the prevention of embolism in atrial fibrillation}

\author{
Carlos Arturo Gómez-Echeverri ${ }^{\mathrm{a}, \mathrm{b}, *}$ y Luis Felipe Ramos-Hurtado ${ }^{\mathrm{a}, \mathrm{b}}$
}

a Unidad de Cardiología, Clínica Medellín, Medellín, Colombia

b Universidad Pontificia Bolivariana, Medellín, Colombia

Recibido el 3 de octubre de 2016; aceptado el 6 de octubre de 2016

Disponible en Internet el 11 de noviembre de 2016

\section{Introducción}

La fibrilación auricular es el principal factor de riesgo de embolia cerebral en personas de edad avanzada ${ }^{1}$. La embolia cerebral secundaria a fibrilación auricular tiene mayor mortalidad e incapacidad que la embolia no relacionada con fibrilación auricular.

Se estima que el $90 \%$ de los eventos embólicos relacionados con fibrilación auricular se originan en la orejuela ${ }^{2}$. La prevención de embolia relacionada con fibrilación auricular se ha basado en el uso de anticoagulación oral con warfarina, la cual reduce el riesgo de ataque cerebrovascular aproximadamente y el de mortalidad de toda causa, en un 64 y $26 \%^{3-7}$. Una proporción importante de pacientes, entre 30 y $50 \%$, no reciben anticoagulación por contraindicaciones o debido a preferencias o temores de pacientes y médicos ${ }^{8,9}$. Por otro lado, el uso de anticoagulantes como prevención secundaria puede disminuir a la mitad, luego de dos años de uso ${ }^{10}$. En años recientes han entrado al mercado nuevos anticoagulantes orales, los cuales tienen una relación riesgo/beneficio adecuada con la warfarina. $\mathrm{El}$ número de pacientes que se inician en el manejo con nuevos

\footnotetext{
* Autor para correspondencia.

Correo electrónico: cargomed@une.net.com (C.A. Gómez-Echeverri).
}

anticoagulantes o se cambian de la warfarina a éstos, sigue en aumento ${ }^{4-6}$; Sin embargo, los costos de estos nuevos medicamentos son altos y su uso es más problemático de lo que se esperaba, como ocurre con el sangrado gastrointestinal ${ }^{11}$. Es claro que los sangrados mayores, incluido el intracerebral, son menores con los nuevos anticoagulantes ${ }^{12,13}$. Por diferentes razones se han intentado diferentes terapias como sustituto de la anticoagulación en pacientes con fibrilación auricular.

\section{Justificación del cierre percutáneo de la orejuela}

La orejuela de la aurícula izquierda es un remanente del desarrollo embrionario de la misma ${ }^{14}$. La orejuela se contrae activamente durante el ritmo sinusal, lo cual está documentado en ecocardiografía transesofágica y resonancia magnética ${ }^{15}$. No obstante, en pacientes con fibrilación auricular, esta capacidad contráctil se pierde y las velocidades del flujo sanguíneo disminuyen, fenómeno que se convierte en estasis sanguínea que incrementa la probabilidad de formación de trombos ${ }^{15,16}$. Es importante resaltar que aunque la presencia de trombos y bajas velocidades en la orejuela se asocia con riesgo embólico, también se ha observado que las placas complejas en la aorta, se relacionan con un aumento del riesgo en mención ${ }^{17}$. 
La fibrilación auricular se divide en fibrilación auricular de origen valvular y fibrilación auricular no valvular. La de origen valvular hace referencia a enfermedad valvular de origen reumático (principalmente estenosis mitral) o presencia de prótesis valvulares. En este grupo de pacientes el riesgo de embolia es muy alto y la única terapia aceptada para su prevención es la warfarina. En el grupo de pacientes con fibrilación auricular de origen no valvular, dicho riesgo se relaciona con otros factores y para determinarlos se usan los sistemas de puntajes $\mathrm{CHADS}_{2}$ y el $\mathrm{CHA}_{2} \mathrm{DS}_{2} \mathrm{VASc}^{18,19}$.

Cada una de las letras significa un factor de riesgo y se asigna un punto a cada uno de ellos, excepto los que se encuentran seguidos del número 2 , lo que significa que ese factor de riesgo confiere el doble de riesgo por sí solo.

El sistema $\mathrm{CHADS}_{2}$ (su sigla en inglés por congestive heart failure) hace referencia a falla cardiaca, hipertensión (hypertension), edad mayor de 75 años (age), diabetes y embolia cerebral (stroke). Las guías europeas de fibrilación auricular de $2010^{20}$ recomendaban que si un paciente no tenía ningún punto se podría elegir entre antiagregación plaquetaria con ácido acetilsalicílico o ningún fármaco, preferiblemente esto último. Si el paciente tenía un punto, podría elegir entre ácido acetilsalicílico o anticoagulación con warfarina, preferiblemente esta última. Si el paciente tenía dos puntos o más debería estar anticoagulado con warfarina.

Posteriormente, apareció el sistema de riesgo $\mathrm{CHA}_{2} \mathrm{DS}_{2}$ VASc, el cual es un poco más complejo, pero intenta definir pacientes de muy bajo riesgo y que no requieren uso de anticoagulación.

El sistema $\mathrm{CHA}_{2} \mathrm{DS}_{2} \mathrm{VASc}$ hace referencia a falla cardiaca (congestive heart failure), hipertensión (hypertension), edad mayor de 75 años (age), diabetes e isquemia cerebral transitoria, embolia cerebral o periférica (stroke), enfermedad vascular carotídea, coronaria o periférica (vascular disease), edad mayor de 65 años (age) y categoría de sexo femenino (sex category). En este sistema se confiere mayor riesgo a tener más de 75 años y se incluyen pacientes mayores de 65 años y pacientes con enfermedad aterosclerótica. Con base en este sistema se dan las siguientes recomendaciones de anticoagulación de la Sociedad europea de cardiología.

\section{Fibrilación auricular de origen valvular}

\section{Anticoagulación oral con warfarina}

\section{Fibrilación auricular no valvular}

- Menores de 65 años sin otros factores de riesgo (incluye mujeres): no requiere terapia antitrombótica.

Si no cumple lo anterior, aplicar el sistema $\mathrm{CHA}_{2} \mathrm{DS}_{2} \mathrm{VASC}$.

- Puntaje 0: no requiere terapia antitrombótica

- Puntaje $\geq 1$ : terapia antitrombótica con los anticoagulantes directos o warfarina.

Pese a que en la actualidad existe consenso respecto al uso de medicamentos anticoagulantes, existe también riesgo de fenómenos hemorrágicos relacionados con el uso de dichos medicamentos. Por esta razón se ha implementado el sistema de riesgo para sangrado asociado a medicamentos anticoagulantes HAS-BLED ${ }^{21}$ que hace referencia a hipertensión (hypertension), función renal o hepática alterada (abnormal renal/liver function), embolia cerebral (stroke), historia de sangrado o predisposición (bleeding history o predisposition), INR lábil (labile INR), anciano (elderly) y consumo concomitante de alcohol o medicamentos (drugs/alcohol concomitantly). Cada uno de estos factores confiere un punto al sistema de riesgo.

Este sistema ha sido validado en varias cohortes y se correlaciona bien con el riesgo de hemorragia intracraneana asociada a anticoagulación. Así, en cada paciente se valora el riesgo hemorrágico y en con $\mathrm{HAS}-\mathrm{BLED} \geq 3$ puntos, se hace un control estricto de la anticoagulación.

En vista de que los pacientes que tienen indicación de anticoagulación pueden tener alto riesgo de sangrado asociado a su terapia, se han intentado algunas técnicas para disminuir el riesgo de embolia originada en la orejuela.

\section{Características de la orejuela izquierda}

Como se expresó anteriormente, la orejuela izquierda, auriculilla o apéndice auricular es un remanente embrionario de la aurícula izquierda. Se ubica en el surco aurículoventricular, en íntima relación con la arteria circunfleja. Tiene una morfología alargada y el ostium puede tener un diámetro de 1 a $4 \mathrm{~cm}$. En su parte interior pueden encontrarse diferentes conformaciones, descritas como "ala de pollo", "cactus", "coliflor" o en "saco de viento",2.

\section{Cierre quirúrgico de la orejuela izquierda}

Desde hace más de 50 años se vienen intentando diferentes técnicas para intentar excluir la orejuela izquierda. Las técnicas quirúrgicas iniciales se llevaban a cabo durante procedimientos de cirugía valvular mitral y MAZE; sin embargo, con frecuencia se reportaban complicaciones hemorrágicas durante el proceso de sutura, debido a la fragilidad del tejido de la orejuela ${ }^{22-25}$. Además, en seguimiento posterior se ha observado cierre incompleto hasta en el $36 \%$ de los casos.

Con base en lo aprendido del cierre quirúrgico de la orejuela y con la convicción de la importancia de su eliminación como fuente de embolia, se han desarrollado diferentes técnicas de cierre de la orejuela.

\section{Cierre percutáneo de la orejuela}

Uno de los primeros dispositivos empleados fue el PLATOO (Percutaneous Left Atrial Transcatheter Occlusion), un dispositivo autoexpandible de nitinol, cubierto con una membrana de polímero. Este se utilizó en algunos estudios multicéntricos, pero su empleo se limitó por la rigidez del dispositivo 26,27 .

Entre las técnicas más relevantes y empleadas en Europa y Estados Unidos están los dispositivos de cierre percutáneo Watchman ${ }^{\circledR}$ y Amplatzer Cardiac Plug ${ }^{\circledR}$. Los dispositivos utilizados actualmente son más flexibles, lo cual favorece su 
adaptación al orificio de la orejuela, que tiene una forma ovalada.

\section{Watchman}

El Watchman es un dispositivo de nitinol en forma de paracaídas con unas pequeñas pestañas alrededor para su fijación dentro de la orejuela. Ha sido el único dispositivo evaluado en dos estudios prospectivos, aleatorizados y controlados y varios estudios observacionales que incluyeron 2.400 pacientes con fibrilación auricular no valvular. El primero y más grande fue el PROTECT-AF (Watchman Left Atrial Appendage System for Embolic Protection in Patients whit Atrial Fibrilation) que incluyó 707 pacientes con fibrilación auricular no valvular, aleatorizados de manera 2:1 para recibir el dispositivo o anticoagulación oral con warfarina ${ }^{28}$. A aquellos que recibieron el dispositivo Watchman se les suministró warfarina al menos por 45 días después del implante. Se realizó ecocardiografía trasesofágica a los 45 días, a los 6 y 12 meses para evaluar flujo residual alrededor del dispositivo. Se descontinuó la warfarina si el cierre de la orejuela era completo o existía un flujo menor de $5 \mathrm{~mm}$. Una vez suspendido este medicamento, los pacientes recibieron anti agregación dual con clopidogrel y ácido acetilsalicílico por seis meses más y posteriormente solo ácido acetilsalicílico. Los pacientes control recibieron warfarina durante todo el estudio.

El PROTECT AF fue un estudio multicéntrico de no inferioridad, en los desenlaces compuestos de ataque cerebro-vascular hemorrágico o isquémico, muerte cardiovascular o inexplicada o embolia sistémica. Tuvo un seguimiento de 3,8 años y se logró éxito del $88 \%$ en el implante del dispositivo. Se logró demostrar que en quienes se implantó el dispositivo hubo una reducción del riesgo relativo en los desenlaces primarios compuestos de ataque cerebro-vascular, embolia y muerte cardiovascular en $40 \%$ (reducción del riesgo absoluto del $1,5 \%$ ) comparado con warfarina.

Los pacientes que recibieron el dispositivo tuvieron menos eventos hemorrágicos cerebrales que el grupo control. Las principales complicaciones observadas en el grupo del dispositivo fueron: derrame pericárdico que requirió intervención (4,8\% de los pacientes), embolización del dispositivo (ocurrió en 3 pacientes de 408 - 0,73\%) y eventos cerebrales embólicos (5 de 408). En un registro posterior al estudio PROTECT-AF llamado CAP Continued Acces Protocol), el cual incluyó 460 pacientes, se osbervó una importante mejoría respecto a seguridad ${ }^{29}$. En este grupo de pacientes el éxito del implante del dispositivo aumentó al $95 \%$, el riesgo de derrame pericárdico fue de 2,2\% y no se documentaron ataques cerebro-vasculares. Esto sugiere, que en la medida que se mejora la experiencia de los operadores, se reduce el riesgo alrededor del procedimiento.

El estudio PREVAIL fue diseñado por el patrocinador en conjunto con la Administración de medicamento y alimentos (FDA) en respuesta a unos cuestionamientos de esta al estudio PROTECT $A F^{30}$. Los pacientes estudiados en el PREVAIL requerían un $\mathrm{CHADS}_{2}$ mayor o igual a 2, para evaluar la efectividad Watchman en una población de relativo alto riesgo de embolia. Para calcular la relación entre la seguridad del procedimiento y el volumen de procedimientos, se exigió que al menos el $20 \%$ de los sitios incluidos en el estudio no tuvieran experiencia previa en el procedimiento. El punto final primario principal era ataque cerebro-vascular (isquémico o hemorrágico), muerte (origen cardiovascular o inexplicada) o embolia sistémica; el otro punto final primario era embolia cerebral o sistémica, ocho días después de la aleatorización y hasta 18 meses después. Se estudiaron 461 personas y fueron aleatorizadas 2:1 entre Watchman y warfarina respectivamente. El PREVAIL falló en demostrar no inferioridad respecto a la terapia con warfarina. De hecho en el grupo Watchman hubo 13 ataques cerebro-vasculares embólicos contra uno en el grupo warfarina.

En la actualidad no existen estudios aleatorizados con el uso del Watchman en pacientes que tengan contraindicación para anticoagulantes.

En marzo de 2015, la FDA aprobó el uso de Watchman en fibrilación auricular no valvular, con indicaciones específicas:

1. Riesgo de ataque cerebro-vascular o embolia sistémica con base en $\mathrm{CHADS}_{2}$ o $\mathrm{CHA}_{2} \mathrm{DS}_{2}$ VASc.

2. Concepto médico para que el paciente reciba warfarina.

3. Criterio claro del paciente para buscar una alternativa no farmacológica para el uso de warfarina, teniendo en cuenta los riesgos y beneficios del dispositivo, comparado con la warfarina.

\section{Amplatzer Cardiac Plug}

Los dispositivos Amplatzer (St Jude Medical) se utilizan desde hace muchos años para la corrección de defectos cardiacos. El Amplatzer para cierre de defecto septal atrial fue modificado para el cierre de la orejuela y recibió el nombre de Amplatzer Cardiac Plug (ACP). El ACP es un dispositivo autoexpandible fabricado con nitinol, que consta de un lóbulo distal y un disco proximal. El lóbulo queda alojado en el interior de la orejuela y posee pestañas para fijarse a las paredes. En la zona proximal el disco cierra el ostium de la orejuela.

El dispositivo ACP tiene en curso un estudio aleatorizado para su aprobación en los Estados Unidos. Hasta la fecha se cuenta con registros, estudios retrospectivos y series de $\operatorname{casos}^{31,32}$. En la actualidad tiene registro para su uso clínico en Europa.

En un estudio de viabilidad y seguridad del dispositivo, se encontró un éxito de implante del $96 \%$, con una tasa de complicaciones del $7 \%{ }^{32}$. A pesar de la menor experiencia del uso del Amplatzer Cardia Plug, el servicio nacional de salud de Gran Bretaña, acepta que ambos dispositivos (Watchman y Amplatzer Cardiac Plug) confieren protección comparable en la prevención de embolia. En la actualidad el ACP está siendo reemplazado por el Amulet, el cual tiene estudios de factibilidad ${ }^{33-35}$ y está disponible para su uso fuera de los Estados Unidos.

En Colombia actualmente se dispone del Watchman, el ACP y el Amulet para el cierre de la orejuela.

\section{Lariat}

Este dispositivo está diseñado para el cierre de la orejuela con un pequeño lazo, el cual se libera con una técnica 
combinada endocavitaria y abordaje pericárdico. Desde el espacio pericárdico se avanza un pequeño lazo hasta la base de la auriculilla y allí se cierra. Tiene aprobación de la FDA ya que se considera una técnica quirúrgica para ligar una estructura con una sutura. El sistema Lariat cuenta también con registro europeo. En Colombia no se dispone en la actualidad de dicho dispositivo. Debido al origen de ser una sutura, no ha tenido estudios que comprueben su eficacia en la prevención de embolia en fibrilación auricular no valvular y solo se cuenta con series de casos. Un estudio de un solo centro reportó una serie de 89 pacientes, en quienes fue posible el cierre en 85 de ellos. Las complicaciones relacionadas con el abordaje fueron de 3,3\% y el uso de anticoagulación posterior al cierre fue del $95 \%$ pese a que el criterio de inclusión era no ser candidato a anticoagulación. La literatura existente no brinda información acerca de la efectividad del Lariat para la prevención de embolia relacionada con la fibrilación auricular.

\section{AtriClip}

Es un dispositivo de titanio recubierto con poliéster para el cierre externo de la orejuela. Inicialmente fue diseñado para el cierre intraoperatorio de otras cirugías cardiacas; sin embargo hay reportes de uso en el abordaje por toracoscopia. En la actualidad no se ha evaluado la efectividad del dispositivo para la prevención de embolia relacionada con fibrilación auricular en estudios clínicos. Este dispositivo se encuentra disponible en nuestro medio y se ha empleado en casos con contraindicación para anticoagulación en quienes por algún motivo no sea posible realizar el cierre percutáneo con uno de los dispositivos con los que se cuenta en la actualidad.

\section{Recomendaciones para el cierre percutáneo de orejuela en pacientes con fibrilación auricular no valvular}

1. Contraindicación para el uso de anticoagulantes orales (coagulopatías, complicación grave por sangrado previo con anticoagulantes) (recomendación I, nivel de evidencia C).

2. Eventos isquémicos recurrentes a pesar de niveles terapéuticos de warfarina o buena adherencia a nuevos anticoagulantes (considerado como falla terapéutica) (recomendación Ilb, nivel de evidencia C).

3. Antecedente de hemorragia intracraneana, ataque cerebro-vascular hemorrágico o microsangrados cerebrales (recomendación I, nivel de evidencia C).

4. Sangrado recurrente sin potencial de solución (angiodisplasia intestinal) (recomendación IIA, nivel de evidencia C).

5. Enfermedad hipertensiva de difícil control y angiopatía cerebral amiloidea (recomendación IIb, nivel de evidencia C).

6. Pacientes con difícil seguimiento para la terapia anticoagulante oral. (recomendación Ilb, nivel de evidencia C).

7. Paciente con antecedente de sangrado de origen no traumático en presencia de anticoagulación con nuevos anticoagulantes o con warfarina en rango terapéutico (recomendación Ila, nivel de evidencia $\mathrm{C}$ ).

8. Preferencia del paciente para el no uso de anticoagulantes orales, conociendo los riesgos y beneficios del dispositivo (Watchman) (recomendación Ila, nivel de evidencia B).

\section{Contraindicaciones}

1. Presencia de trombo en orejuela izquierda determinado por ecocardiograma transesofágico.

2. Paciente con comorbilidades importantes y expectativa de vida inferior a un año.

3. Pacientes con enfermedad reumática cardíaca.

\section{Conclusión}

La fibrilación auricular es la arritmia sostenida más común en la práctica clínica y es el principal factor de riesgo para embolia cerebral en personas de edad avanzada, con una alta morbilidad y mortalidad. Además, por su alta prevalencia tiene un incremento de costos de atención para los sistemas de salud. Diferentes estudios han demostrado que el $90 \%$ de los trombos relacionados con fibrilación auricular se originan en la orejuela izquierda. La prevención de embolia asociada a fibrilación auricular se ha llevado a cabo con medicamentos anticoagulantes como los antagonistas de la vitamina $\mathrm{K}$ y en los últimos años con el uso de los nuevos anticoagulantes. Sin embargo, existe un número importante de pacientes que no pueden recibir dichos medicamentos por contraindicaciones o complicaciones derivadas de su uso. Por tal motivo se han buscado alternativas para la prevención de embolia con la oclusión de la orejuela por diferentes vías, tanto quirúrgica como percutánea.

\section{Conflicto de intereses}

Los autores declaran no tener ningún conflicto de intereses.

\section{Bibliografía}

1. Roger VL, Go AS, Lloyd-Jones DM, et al. Heart disease and stroke statistics-2011 update: a report from the American Heart Association. Circulation. 2011;123:e18-209.

2. Blackshear JL, Odell JA. Appendage obliteration to reduce stroke in cardiac surgical patients with atrial fibrillation. Ann Thorac Surg. 1996;61:755-9.

3. Hart RG, Pearce LA, Aguilar MI. Meta-analysis: antithrombotic therapy to prevent stroke in patients who have nonvalvular atrial fibrillation. Ann Intern Med. 2007;146:857-67.

4. Camm AJ, Lip GYH, De Caterina R, et al. Focused update of the ESC Guidelines for the management of atrial fibrillation. Eur Heart J. 2012;33:2719-47.

5. Pillarisetti J, Lakkireddy D. Atrial fibrillation in Europe: state of the state in disease management. Eur Heart J. 2014;35:3326-7.

6. Cocco G, Jerie P. New concepts in the therapy of atrial fibrillation. Cardiology J. printed on line. 2015-08-26.

7. Lipp GYH. The CHA2DS2VASc score for stroke risk stratification in patients with atrial fibrillation: a brief history. Eur Heart J. 2015;36:2880-5. 
8. Bungard TJ, Ghali WA, Teo KK, et al. Why do patients with atrial fibrillation not receive warfarin? Arch Intern Med. 2000;160:41-6.

9. Nieuwlaat R, Capucci A, Camm AJ, et al. Atrial fibrillation management: a prospective survey in ESC Member Countries: The Euro Heart Survey on Atrial Fibrillation. Eur Heart J. 2005;26:2422-34.

10. Glader EL, Sjolander M, Eriksson M, et al. Persistent use of secondary preventive drugs declines rapidly during the first 2 years after stroke. Stroke. 2010;41:397-401.

11. Shurlock B. New oral anticoagulants for non-valvular atrial fibrillation: harder to handle than expected? Eur Heart J. 2014;35:1825-30.

12. Bonetti N, Laube ES, Beer JH. NOACs: "NO Anticoagulant without Consideration'”. Cardiovasc Med. 2014;17:213-20.

13. Camm AJ, Amarenco P, Haas S, et al. XATUS: a real-world, prospective, observational study of patients treated with rivaroxaban for stroke prevention in atrial fibrillation. Eur Heart J. 2016;37:1145-53.

14. Al-Saady NM, Obel OA, Camm AJ. Left atrial appendage: structure, function, and role in thromboembolism. Heart. 1999;82:547-54.

15. Pollick C, Taylor D. Assessment of left atrial appendage function by transesophageal echocardiography. Implications for the development of thrombus. Circulation. 1991;84:223-31.

16. Mugge A, Kuhn H, Nikutta $P$, et al. Assessment of left atrial appendage function by biplane transesophageal echocardiography in patients with nonrheumatic atrial fibrillation: identification of a subgroup of patients at increased embolic risk. J Am Coll Cardiol. 1994;23:599-607.

17. Zabalgoitia M, Halperin JL, Pearce LA, et al. Transesophageal echocardiographic correlates of clinical risk of thromboembolism in nonvalvular atrial fibrillation. Stroke Prevention in Atrial Fibrillation III Investigators. J Am Coll Cardiol. 1998;31: 1622-6.

18. Gage BF, Waterman AD, Shannon W, et al. Validation of clinical classification schemes for predicting stroke: results from the National Registry of Atrial Fibrillation. JAMA. 2001;285:2864-70.

19. Lip GY, Nieuwlaat R, Pisters R, et al. Refining clinical risk stratification for predicting stroke and thromboembolism in atrial fibrillation using a novel risk factor-based approach: the Euro Heart Survey on Atrial Fibrillation. Chest. 2010;137:263-72.

20. Camm AJ, Kirchhof P, Lip GY, et al. Guidelines for the management of atrial fibrillation: the Task Force for the Management of Atrial Fibrillation of the European Society of Cardiology (ESC). Europace. 2010;12:1360-420.

21. Lip GY, Frison L, Halperin JL, et al. Comparative validation of a novel risk score for predicting bleeding risk in anticoagulated patients with atrial fibrillation: the HAS-BLED (Hypertension, Abnormal renal/liver function, Stroke, Bleeding history or predisposition, Labile INR, Elderly, Drugs/alcohol concomitantly) score. J Am Coll Cardiol. 2011;57:173-80.
22. Chatterjee S, Alexander JC, Pearson PJ, et al. Left atrial appendage occlusion: lessons learned from surgical and transcatheter experiences. Ann Thorac Surg. 2011;92:2283-92.

23. DiSesa VJ, Tam S, Cohn LH. Ligation of the left atrial appendage using an automatic surgical stapler. Ann Thorac Surg. 1988;46:652-3.

24. Odell JA, Blackshear JL, Davies E, et al. Thoracoscopic obliteration of the left atrial appendage: potential for stroke reduction? Ann Thorac Surg. 1996;61:565-9.

25. Pennec PY, Jobic Y, Blanc JJ, et al. Assessment of different procedures for surgical left atrial appendage exclusion. Ann Thorac Surg. 2003;76:2168-9.

26. Ostermayer SH, Reisman M, Kramer PH, et al. Percutaneous Left Atrial Appendage Transcatheter Occlusion (PLAATO system) to prevent stroke in high-risk patients with non-rheumatic atrial fibrillation: results from the international multi-center feasibility trials. J Am Coll Cardiol. 2005;46:9-14.

27. Bayard YL, Omran H, Neuzil P, et al. PLAATO (Percutaneous Left Atrial Appendage Transcatheter Occlusion) for prevention of cardioembolic stroke in non-anticoagulation eligible atrial fibrillation patients: results from the European PLAATO study. Eurolntervention. 2010;6:220-6.

28. Holmes DR, Reddy VY, Turi ZG, et al. Percutaneous closure of the left atrial appendage versus warfarin therapy for prevention of stroke in patients with atrial fibrillation: a randomised noninferiority trial. Lancet. 2009;374:534-42.

29. Reddy VY, Holmes D, Doshi SK, et al. Safety of percutaneous left atrial appendage closure: results from the Watchman Left Atrial Appendage System for Embolic Protection in Patients with AF (PROTECT AF) clinical trial and the Continued Access Registry. Circulation. 2011;123:417-24.

30. Holmes DR Jr, Kar S, Price MJ, et al. Prospective randomized evaluation of the Watchman left atrial appendage closure device in patients with atrial fibrillation versus long-term warfarin therapy: the PREVAIL trial. J Am Coll Cardiol. 2014;64:1-12.

31. Urena M, Rodes-Cabau J, Freixa X, et al. Percutaneous left atrial appendage closure with the AMPLATZER cardiac plug device in patients with nonvalvular atrial fibrillation and contraindications to anticoagulation therapy. J Am Coll Cardiol. 2013;62:96-102.

32. Park JW, Bethencourt A, Sievert $H$, et al. Left atrial appendage closure with Amplatzer cardiac plug in atrial fibrillation: initial European experience. Catheter Cardiovasc Interv. 2011;77:700-6.

33. Whisenant B, Weiss P. Left atrial appendage closure with transcatheter-delivered devices. Intervent Cardiol Clin. 2014;3:209-18.

34. Freixa X, Tzikas A, Sobrino A, et al. Left atrial appendage closure with the Amplatzer Cardiac Plug: impact of shape and device sizing on follow-up leaks. Int J Cardiol. 2013;168:1023-7.

35. Freixa X, Chan JL, Tzikas A, et al. The Amplatzer Cardiac Plug 2 for left atrial appendage occlusion: novel features and firstin-man experience. Eurolntervention. 2013;8:1094-8. 